Article

\title{
Synthesis of Chiral Chalcone Derivatives Catalyzed by the Chiral Cinchona Alkaloid Squaramide
}

\section{Dandan Xie ${ }^{\dagger}$, Ying Xie ${ }^{\dagger}$, Yan Ding, Jian Wu and Deyu Hu *}

State Key Laboratory Breeding Base of Green Pesticide and Agricultural Bioengineering, Key Laboratory of Green Pesticide and Agricultural Bioengineering, Ministry of Education, Guizhou University, Huaxi District, Guiyang 550025, China; E-Mails: xddxed@163.com (D.X.); xieying2045@163.com (Y.X.); diding1011@163.com (Y.D.); wujian2691@126.com (J.W.)

$\dagger$ These authors contributed equally to this work.

* Author to whom correspondence should be addressed; E-Mail: fcc.dyhu@gzu.edu.cn; Tel./Fax: +86-851-829-2170.

External Editor: Derek J. McPhee

Received: 3 November 2014; in revised form: 13 November 2014 / Accepted: 13 November 2014 / Published: 25 November 2014

\begin{abstract}
An effective method has been developed for the preparation of novel chiral chalcone derivatives under mild conditions from the easily accessible starting materials nitromethane and chalcone derivatives 2 . The corresponding products were obtained in moderate yields with excellent enantioselectivities (up to 99\%).
\end{abstract}

Keywords: chalcone derivatives; enantioselective catalysis; chiral cinchona alkaloid squaramides; addition reaction

\section{Introduction}

Chalcones are not only excellent scaffolds for synthetic manipulations but also possess multiple biological and medicinal properties as antioxidant [1], antibacterial [2-4], antifungal [5], anti-Alzheimer's disease [6], anticancer [7], antitumor [8], antimalarial [9], antiproliferative [10], anti-inflammatory [11], and anti-HIV-1 agents [12]. Research laboratories worldwide are focusing on the synthesis of different chalcone analogues for the development of novel and potent drugs [13]. Asymmetric catalysis has proven its potential in numerous demanding applications which have been 
reported and developed for the direct asymmetric addition reactions of chalcones in the past. Lu et al. reported a highly diastereoselective and enantioselective conjugate addition of phthalide derivatives to chalcones, leading to the formation of chiral phthalides bearing vicinal quaternary and tertiary stereogenic centers [14]. Previous work using chiral squaramide-based organ catalysts in similar enantioselective Michael additions have been reported [15,16], and asymmetric cyclopropanation of chalcones using bromomalonates as the nucleophiles in a Michael-Initiated Ring Closing reaction was developed by Waser [17]. The reaction performed well for electron neutral and electron deficient chalcones, giving the products in yields of up to $98 \%$ and with enantiomeric ratios up to 91:9. Surprisingly, the use of chiral squaramide catalysts and nitromethane to facilitate addition reactions was described only a few times. Based on this concept and the knowledge gathered therein we surmised that some catalyst structures Q1-Q4 might be efficient in the direct addition reactions between nitromethane (1) with chalcone derivatives 2 (Figure 1). Herein we described our work toward preparation of such interesting chiral moieties in the presence of a variety of known chiral cinchona alkaloid squaramides (Scheme 1).

Figure 1. The catalysts surveyed in the work.

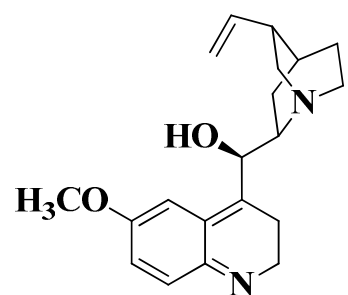

Q 1<smiles>COc1ccc2nccc([C@H](N)CN3CCC4CCC3C4)c2c1</smiles>

Q 2

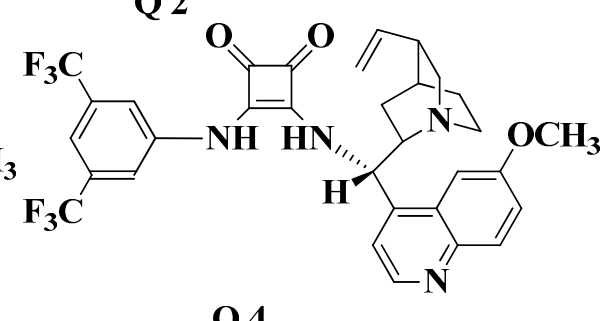

Q 4

Scheme 1. Synthesis of Chiral Chalcone Derivatives 3a-i.

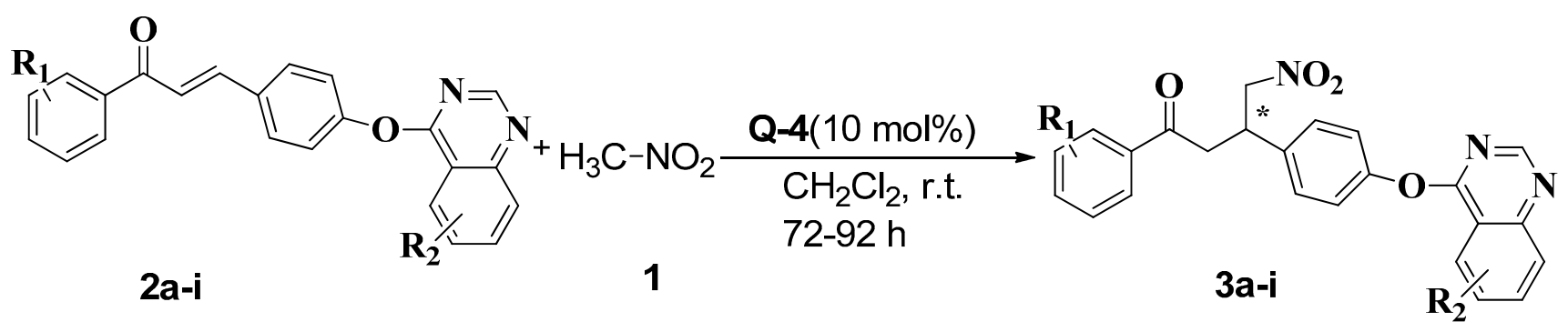

\section{Results and Discussion}

\subsection{Chiral Catalysts Screening}

As shown in Figure 1, various catalysts including cinchona alkaloids Q1 and Q2, and cinchona alkaloid-derived squaramides Q3 and Q4 were developed and surveyed in the reaction. All the 
catalysts surveyed in the work ubiquitously formed the chiral product $\mathbf{3 g}\left(\mathrm{R}_{1}=4-\mathrm{Cl}, \mathrm{R}_{2}=6-\mathrm{Me}\right)$. The results are summarized in Table1. (E)-1-(4-Chlorophenyl)-3-(4-((6-methylquinazolin-4-yl)oxy)phenyl) prop-2-en-1-one $(\mathbf{2 g})$ and nitromethane (1) were adopted as the starting materials for the initial exploration of the asymmetric addition reaction. The reaction was carried out in dichloromethane in the presence of $10 \mathrm{~mol} \%$ catalyst at room temperature for $72 \mathrm{~h}$. Using catalyst Q1, the product $\mathbf{3 g}$ was obtained in 60\% ee (Table 1, entry 1). As for the other catalysts, Q2 and Q3 afforded lower yield and moderate enantioselectivity (Table 1, entry 2 and entry 3, 45\% and 70\% ee, respectively) in the same solvent. Higher ee values were obtained in dichloromethane with catalyst Q4 (Table 1, entries 4, 96\%). Cinchona alkaloid-derived squaramide Q4 gave excellent enantioselectivity with moderate yield.

Table 1. Catalyst screening studies ${ }^{[a]}$.

\begin{tabular}{ccccc}
\hline Entry & Catalyst & Solvent & Yield [\%] $^{[\mathbf{b}]}$ & $\boldsymbol{e e}\left[\right.$ [\%] $^{[\mathbf{c}]}$ \\
\hline 1 & Q1 & $\mathrm{CH}_{2} \mathrm{Cl}_{2}$ & 20.2 & 60 \\
2 & Q2 & $\mathrm{CH}_{2} \mathrm{Cl}_{2}$ & 35.0 & 45 \\
3 & Q3 & $\mathrm{CH}_{2} \mathrm{Cl}_{2}$ & 37.2 & 70 \\
4 & Q4 & $\mathrm{CH}_{2} \mathrm{Cl}_{2}$ & 46.0 & 96 \\
\hline
\end{tabular}

[a] Unless otherwise indicated, all reactions were conducted with $1(0.9 \mathrm{mmol}), \mathbf{2 g}(0.45 \mathrm{mmol})$ and the catalyst $(10 \mathrm{~mol} \%, 0.045 \mathrm{mmol})$ in dichloromethane $(4 \mathrm{~mL})$ at room temperature for $72 \mathrm{~h}$. ${ }^{[b]}$ Isolated yield after chromatographic purification. ${ }^{[c]}$ Determined by HPLC analysis (ChiralpakAD-H).

Table 2. Optimization studies ${ }^{[a]}$.

\begin{tabular}{ccccc}
\hline Entry & Temperature $\left[{ }^{\circ} \mathbf{C}\right]$ & Solvent & Yield [\%] ${ }^{[\mathbf{b}]}$ & $\boldsymbol{e e ~ [ \% ] ~}{ }^{\left[{ }^{[\mathbf{c}}\right.}$ \\
\hline 1 & $\mathrm{rt}$ & $\mathrm{CH}_{2} \mathrm{Cl}_{2}$ & 46.2 & 96 \\
2 & $\mathrm{rt}$ & $\mathrm{EtOH}$ & 30.0 & 50 \\
3 & $\mathrm{rt}$ & $\mathrm{PhCH}_{3}$ & 40.9 & 67 \\
4 & 60 & $\mathrm{PhCH}_{3}$ & 60.2 & 55 \\
5 & 0 & $\mathrm{CH}_{2} \mathrm{Cl}_{2}$ & 46.0 & 79 \\
6 & 40 & $\mathrm{CH}_{2} \mathrm{Cl}_{2}$ & 50.2 & 85 \\
7 & $\mathrm{rt}^{[\mathrm{d}]}$ & $\mathrm{CH}_{2} \mathrm{Cl}_{2}$ & 40.2 & 88 \\
8 & $\mathrm{rt}^{[\mathrm{e}]}$ & $\mathrm{CH}_{2} \mathrm{Cl}_{2}$ & 55.2 & 97 \\
\hline
\end{tabular}

[a] Unless otherwise indicated, all reactions were conducted with 1 (0.9 mmol), $\mathbf{2 g}(0.45 \mathrm{mmol})$ and Q4 catalyst $(10 \mathrm{~mol}-\%, 0.045 \mathrm{mmol})$ in the different solvents $(4 \mathrm{~mL})$ at different temperatures for $72 \mathrm{~h}$.

${ }^{[b]}$ Isolated yield after chromatographic purification. ${ }^{[c]}$ Determined by HPLC analysis (Chiral pakAD-H).

${ }^{[\mathrm{d}]}$ Reaction time: 42 h. ${ }^{[\mathrm{e}]}$ Reaction time: $100 \mathrm{~h}$.

\subsection{Optimization Studies}

To optimize the reaction regime, the synthesis of chiral compound (-)-3g was carried out under several conditions. The effects of different solvents, reaction time and temperature were investigated using Q4 as catalyst; the results are shown in Table 2. From the data presented in the table it can be concluded that dichloromethane appeared to be the best solvent to obtain high ee values; other solvents provided much poorer enantioselectivities ranging between 50\%-67\% ee (Table 2, entry 1, entry 2 and entry 3). Moreover, the effects of reaction temperature and time on the addition reaction were also investigated (Table 2, entries 5-7). When the reaction time was prolonged from 42 to $72 \mathrm{~h}$, the ee 
value of (-)-3g was increased from $88 \%$ to $96 \%$ (Table 2, entries 4 and 6). Extending the reaction time up to $100 \mathrm{~h}$ resulted in only a tiny improvement of the ee value (97\%, Table 2 , entry 8$)$ if compared with that obtained after $72 \mathrm{~h}$ of reaction ( $96 \%$, Table 2 , entry 4$)$. As for the reaction temperature, it could be observed that when the reaction temperature was increased from $0{ }^{\circ} \mathrm{C}$ to room temperature and $40{ }^{\circ} \mathrm{C}$, the ee values of (-)-3g were $79 \%, 96 \%$ and $85 \%$, respectively (Table 2 , entries $1,5-6$ ). Hence, the optimal reaction conditions were selected as following: $\mathrm{CH}_{2} \mathrm{Cl}_{2}$ as a solvent, room temperature, $72 \mathrm{~h}$ reaction time, $\mathrm{Q} 4$ as a catalyst.

\subsection{Synthesis of Chiral Chalcones Derivatives (-)-3a-i}

The optimized protocol was then expanded to a wide variety of chalcone derivatives and the results are summarized in Table 3. The relationships of the enantioselectivity to different $R_{1}$ and $R_{2}$ values were observed.

Table 3. Synthesis of chiral chalcones derivatives (-)-3a-i ${ }^{[a]}$.

\begin{tabular}{ccccc}
\hline Compounds & $\mathbf{R}_{\mathbf{1}}$ & $\mathbf{R}_{\mathbf{2}}$ & Yield [\%] $^{[\mathbf{b}]}$ & $\boldsymbol{e e}[\mathbf{\%}]^{[\mathbf{c}]}$ \\
\hline$(-)-3 \mathrm{a}$ & $\mathrm{H}$ & $8-\mathrm{Me}$ & 45.7 & 81.4 \\
$(-)-3 \mathrm{~b}$ & $2,4-\mathrm{diCl}$ & $8-\mathrm{Me}$ & 40.0 & 91.5 \\
$(-)-3 \mathrm{c}$ & $\mathrm{H}$ & $6-\mathrm{Me}$ & 40.0 & 86.0 \\
$(-)-3 \mathrm{~d}$ & $2,4-\mathrm{diCl}$ & $6-\mathrm{Me}$ & 38.0 & 91.8 \\
$(-)-3 \mathrm{e}$ & $4-\mathrm{Cl}$ & $\mathrm{H}$ & 35.0 & 99.0 \\
$(-)-3 \mathrm{f}$ & $4-\mathrm{MeO}$ & $\mathrm{H}$ & 42.2 & 92.0 \\
$(-)-3 \mathrm{~g}$ & $4-\mathrm{Cl}$ & $6-\mathrm{Me}$ & 46.1 & 95.9 \\
$(-)-3 \mathrm{~h}$ & $2-\mathrm{Cl}$ & $6-\mathrm{Me}$ & 40.3 & 96.0 \\
$(-)-3 \mathrm{i}$ & 2-F & 6-Me & 42.5 & 92.5 \\
\hline
\end{tabular}

[a] Unless otherwise indicated, all reactions were conducted with $\mathbf{2 a - i}(0.45 \mathrm{mmol}), \mathbf{1}(0.9 \mathrm{mmol})$ and the Q4 catalyst $(10 \mathrm{~mol}-\%, 0.045 \mathrm{mmol})$ in $\mathrm{CH}_{2} \mathrm{Cl}_{2}(4 \mathrm{~mL})$ at room temperature for $72 \mathrm{~h}$. ${ }^{[\mathrm{b}]}$ Isolated yield after chromatographic purification. ${ }^{[c]}$ Determined by HPLC analysis (Chiralpak AD-H).

The data indicated that compounds (-)-3e, (-)-3g, and (-)-3h exhibited higher enantioselectivity than other compounds, with ee values of $99.0 \%, 95.9 \%$, and $96.0 \%$, respectively. When $\mathrm{R}_{1}$ was $\mathrm{H}$, 2-Cl, 4-Cl or 2-F and $\mathrm{R}_{2}$ was $\mathrm{H}$ or 6-Me the corresponding target chiral chalcones exhibited excellent enantioselectivity. The strongest enantioselectivity was observed when $\mathrm{R}_{1}$ was $4-\mathrm{Cl}, 2-\mathrm{Cl}$ and $\mathrm{R}_{2}$ was substituted with H, 6-Me- groups. Compared with (-)-3h, the target compounds (-)-3b, (-)-3d, (-)-3f, and (-)-3i also displayed good enantioselectivity, with ee values of $91.5 \%, 91.8 \%, 92.0 \%$ and $92.5 \%$, respectively. The presence of $4-\mathrm{Cl}$ or 2-Cl atoms in a benzene moiety and 6-Me group or $\mathrm{H}$-atom in a quinazoline ring of chalcones $\mathbf{2}$ did not significantly affect the reactivity and enantioselectivity of the reaction. $\mathrm{An} H$ atom as $\mathrm{R}_{1}$ and $6-\mathrm{Me}$ or $8-\mathrm{Me}$ as $\mathrm{R}_{2}$ in substrates 2 had some effect on the enantioselectivity of the addition reactions; compounds (-)-3a and (-)-3c afforded moderate enantioselectivity with ee values of $81.4 \%$ and $86.0 \%$, respectively. 


\section{Experimental Section}

\subsection{General Information}

Unless otherwise stated, all the reagents and reactants were purchased from commercial suppliers; melting points were uncorrected and determined on a XT-4 binocular microscope (Beijing Tech Instrument Co., Beijing, China). The ${ }^{1} \mathrm{H}-\mathrm{NMR}$ and ${ }^{13} \mathrm{C}$-NMR spectra were recorded on an ECX 500 NMR spectrometer (JEOL, Ltd., Tokoy, Japan) at room temperature operating at $500 \mathrm{MHz}$ for ${ }^{1} \mathrm{H}-\mathrm{NMR}$ and $125 \mathrm{MHz}$ for ${ }^{13} \mathrm{C}-\mathrm{NMR}$, using $\mathrm{CDCl}_{3}$, or DMSO- $d_{6}$ as solvents and TMS as an internal standard; infrared spectra were recorded in $\mathrm{KBr}$ on a VECTOR 22 spectrometer (Bruker Ltd., Ettlingen, German); mass spectral studies were conducted on an Agilent 5973 organic mass spectrometer (Agilent Technologies Inc., Santa Clara, CA, USA). The course of the reactions was monitored by TLC; analytical TLC was performed on silica gel $\mathrm{GF}_{254}$ plates; column chromatographic purification was carried out using silicagel. The enantiomeric excess was determined by HPLC using a Chiral pak AD-H column.

\subsection{Preparation of Chiral Catalyst $\mathbf{Q} 4$}

3-(3,5-Ditrifluoromethylphenylamino)4-methoxybutane-3-en-1,2-dione (1.0 mmol) was slowly added with stirring to a mixture of dichloromethane $(4 \mathrm{~mL})$ and 9-aminodeoxyquinine (1.02 mmol). After completion of the addition, the stirring was continued for $12 \mathrm{~h}$ at room temperature. The mixture was concentrated, and the crude product was purified by preparative TLC with a mixture of petroleum ether and ethyl acetate $(\mathrm{V}: \mathrm{V}=1: 5)$ as developing solvent to give chiral catalyst Q4. Yield, 56.8\%; m.p. $170-171{ }^{\circ} \mathrm{C},[\alpha]_{D}^{25}=+70.2\left(\mathrm{c}=0.52\right.$, DMSO) (lit. $[18,19]$, m.p. $181-183{ }^{\circ} \mathrm{C},[\alpha]_{D}^{25}=+65.1$ $(c=0.55, \mathrm{DMSO}))$.

\subsection{Preparation of Intermediates $\mathbf{2}$}

4-Hydroxychalcone (3 mmol), 4-chloroquinazoline (3 mmol), $\mathrm{K}_{2} \mathrm{CO}_{3}(6.3 \mathrm{mmol})$, and acetone $(15 \mathrm{~mL})$ were added to an oven-dried one-neck $50 \mathrm{~mL}$ round-bottom flask equipped with a magnetic stirring bar. The resulting mixture was stirred at $40{ }^{\circ} \mathrm{C}$ for $10 \mathrm{~h}$, poured into ice water $(40 \mathrm{~mL})$, and then separated. The aqueous phase was acidified with $10 \% \mathrm{HCl}$ to $\mathrm{pH} 5-7$ and then filtered. The residue was dried and recrystallized from ethanol to obtain compounds $\mathbf{2 a - i}$ as white solids [20].

\subsection{Preparation of Title Chiral Compounds (-)-3a-3i}

To a well stirred solution of nitromethane $(0.9 \mathrm{mmol})$ and chalcone derivative $2(0.45 \mathrm{mmol})$ in $4 \mathrm{~mL}$ of dichloromethane, Q4 $(0.045 \mathrm{mmol})$ was added. The resulting mixture was stirred at room temperature and monitored by TLC. After stirring for $72 \mathrm{~h}$, the mixture was concentrated, and the crude product was purified by preparative TLC with a mixture of petroleum ether and ethyl acetate $(\mathrm{V}: \mathrm{V}=2: 1)$ as developing solvent to give title chiral compounds (-)-3a-3i.

(-)-3-(4-((8-Methylquinazolin-4-yl)oxy)phenyl)-4-nitro-1-phenylbutan-1-one [(-)-3a]: Light brown solid; yield $45.7 \%$, m.p. $133-135^{\circ} \mathrm{C} ;[\alpha]_{D}^{25}=-62.4\left(\mathrm{c}=1.01, \mathrm{CHCl}_{3}\right)$; IR $\left(\mathrm{KBr}, \mathrm{cm}^{-1}\right)$ v: $3442.8(\mathrm{C}-\mathrm{H}$, 
$\mathrm{Qu}-\mathrm{N}=\mathrm{CH}-\mathrm{N}=\mathrm{C}), \quad 1681.3(\mathrm{C}=\mathrm{O}), 1610.8(\mathrm{C}=\mathrm{N}), 1592.3-1485.4 \quad(\mathrm{C}=\mathrm{C}$, benzene and Qu-ring $)$; ${ }^{1} \mathrm{H}-\mathrm{NMR}$ (DMSO-d6, ppm): $\delta 8.74(\mathrm{~s}, 1 \mathrm{H}, \mathrm{Qu}-2-\mathrm{H}), 8.18$ (d, 1H, Qu-7-H, $\left.J=9.05 \mathrm{~Hz}\right), 7.98(\mathrm{~d}, 2 \mathrm{H}$, Ar'-6-H, $J=7.45$ Hz), 7.89 (d, 1H, Qu-5-H, $J=6.85$ Hz), 7.66 (t, 2H, Ar-2-H, Ar-6-H, $J_{1}=8.00 \mathrm{~Hz}$, $J_{2}=7.45 \mathrm{~Hz}$ ), 7.56-7.51 (m, 4H, Ar'-3-H, Ar'-4-H, Ar'-5-H, Qu-6-H),7.29 (d, 2H, Ar-3-H, Ar-5-H, $J=8.60 \mathrm{~Hz}), 5.06-4.91\left(\mathrm{~m}, 2 \mathrm{H},-\mathrm{CH}_{2}-\mathrm{NO}_{2}\right), 4.17-4.11(\mathrm{~m}, 1 \mathrm{H}, \mathrm{CH}), 3.69-3.55\left(\mathrm{~m}, 2 \mathrm{H},-\mathrm{CH}_{2}-\mathrm{CO}\right)$, $2.68\left(\mathrm{~s}, 3 \mathrm{H}, \mathrm{CH}_{3}\right) ;{ }^{13} \mathrm{C}-\mathrm{NMR}\left(\mathrm{DMSO}-d_{6}, \mathrm{ppm}\right): \delta 197.92,167.01,153.57,151.85,150.64,138.08$, 136.88, 136.31, 134.92, 134.02, 129.69, 129.32, 128.52, 128.10, 122.60, 121.45, 116.00, 80.18, 41.74, 39.18, 17.68. MS (ESI) $m / z: 428.3\left([\mathrm{M}+\mathrm{H}]^{+}\right), 450.3\left([\mathrm{M}+\mathrm{Na}]^{+}\right) ; 81.42 \%$ ee as determined by HPLC (Daicel Chiralpak AD-H, hexane/ethanol $=50: 50$, flow rate $1.0 \mathrm{~mL} / \mathrm{min}, \lambda=254 \mathrm{~nm}), \mathrm{t}_{R}($ minor $)=$ $33.16 \mathrm{~min}, \mathrm{t}_{R}$ (major) $=37.68 \mathrm{~min}$.

(-)-1-(2,4-Dichlorophenyl)-3-(4-((8-methylquinazolin-4-yl)oxy)phenyl)-4-nitrobutan-1-one [(-)-3b]: Light yellow solid; yield $40.0 \%$, m.p. $65-67^{\circ} \mathrm{C}$; $[\alpha]_{D}^{25}=-86.4\left(\mathrm{c}=0.96, \mathrm{CHCl}_{3}\right)$; IR $\left(\mathrm{KBr}, \mathrm{cm}^{-1}\right) v$ : $3445.2(\mathrm{C}-\mathrm{H}, \mathrm{Qu}-\mathrm{N}=\mathrm{CH}-\mathrm{N}=\mathrm{C}), 1697.4(\mathrm{C}=\mathrm{O}), 1614.4(\mathrm{C}=\mathrm{N}), 1581.6-1497.4(\mathrm{C}=\mathrm{C}$, benzene and Qu-ring), $771.53(\mathrm{C}-\mathrm{Cl}) \mathrm{cm}^{-1}$; ${ }^{1} \mathrm{H}-\mathrm{NMR}$ (DMSO-d6, ppm): $\delta 8.76(\mathrm{~s}, 1 \mathrm{H}, \mathrm{Qu}-2-\mathrm{H}), 8.19$ (d, 1H, Ar'-3-H, $J=8.00 \mathrm{~Hz}$ ), 7.89 (d, 1H, Ar'-3-H, $J=7.45 \mathrm{~Hz}$ ), 7.70 (s, 1H, Qu-7-H), 7.67 (d, 2H, Ar'-5-H, Qu-6-H, $J=8.05 \mathrm{~Hz}), 7.57$ (d, 1H, Qu-5-H, $J=6.85 \mathrm{~Hz}), 7.49$ (d, 2H, Ar'-2-H, Ar'-6-H, $J=8.60 \mathrm{~Hz}), 7.29$ (d, $2 \mathrm{H}, \mathrm{Ar}-3-\mathrm{H}, \mathrm{Ar}-5-\mathrm{H}, J=8.00 \mathrm{~Hz}), 5.04-4.92$ (m, 2H, $\left.-\mathrm{CH}_{2}-\mathrm{NO}_{2}\right), 4.08-4.03$ (m, 1H, CH), $3.52(\mathrm{~d}, 2 \mathrm{H}$, $-\mathrm{CH}_{2}-\mathrm{CO}, J=6.85 \mathrm{~Hz}$ ), 2.69 (s, 3H, $\left.\mathrm{CH}_{3}\right) ;{ }^{13} \mathrm{C}-\mathrm{NMR}$ (DMSO-d6, ppm): $\delta 199.45,167.06,153.57$, 151.96, 150.71, 137.46, 137.23, 136.83, 136.31, 134.91, 131.68, 131.68, 131.22, 130.64, 129.72, 128.17, 128.10, 122.66, 121.46, 115.92, 79.84, 45.59, 39.29, 17.69. MS (ESI) $m / z: 496.2\left([\mathrm{M}+\mathrm{H}]^{+}\right), 518.2$ $\left([\mathrm{M}+\mathrm{Na}]^{+}\right) ; 91.45 \%$ ee as determined by HPLC (Daicel Chiralpak AD-H, hexane/ethanol = 50:50, flow rate $1.0 \mathrm{~mL} / \mathrm{min}, \lambda=254 \mathrm{~nm}), \mathrm{t}_{R}($ minor $)=24.49 \mathrm{~min}, \mathrm{t}_{R}($ major $)=27.49 \mathrm{~min}$.

(-)-3-(4-((6-Methylquinazolin-4-yl)oxy)phenyl)-4-nitro-1-phenylbutan-1-one [(-)-3c]: White solid; yield $40.0 \%$, m.p. $190-102{ }^{\circ} \mathrm{C} ;[\alpha]_{D}^{25}=-78.4\left(\mathrm{c}=0.78, \mathrm{CHCl}_{3}\right)$; IR $\left(\mathrm{KBr}, \mathrm{cm}^{-1}\right)$ v: $3450.2(\mathrm{C}-\mathrm{H}$, $\mathrm{Qu}-\mathrm{N}=\mathrm{CH}-\mathrm{N}=\mathrm{C}), \quad 1685.8 \quad(\mathrm{C}=\mathrm{O}), \quad 1653.0 \quad(\mathrm{C}=\mathrm{N}), \quad 1588.1-1498.7 \quad(\mathrm{C}=\mathrm{C}$, benzene and Qu-ring $)$; ${ }^{1} \mathrm{H}-\mathrm{NMR}$ (DMSO-d6, ppm): $\delta 8.66(\mathrm{~s}, 1 \mathrm{H}, \mathrm{Qu}-2-\mathrm{H}), 8.13$ (s, 1H, Qu-4-H), 7.98 (d, 2H, Qu-7-H, Qu-8-H, $J=8.05$ Hz), 7.91-7.87 (m, 2H, Ar'-2-H, Ar'-6-H), 7.66 (t, 1H, Ar'-4-H, $J_{1}=6.90 \mathrm{~Hz}$, $J_{2}=7.40 \mathrm{~Hz}$ ), 7.56-7.52 (m, 4H, Ar'-3-H, Ar'-5-H, Ar-2-H, Ar-6-H), 7.29 (d, 2H, Ar-3-H, Ar-5-H, $J=6.90 \mathrm{~Hz}), 5.06-4.91\left(\mathrm{~m}, 2 \mathrm{H},-\mathrm{CH}_{2}-\mathrm{NO}_{2}\right), 4.16-4.10(\mathrm{~m}, 1 \mathrm{H}, \mathrm{CH}), 3.68-3.55\left(\mathrm{~m}, 2 \mathrm{H},-\mathrm{CH}_{2}-\mathrm{CO}\right)$, $2.56\left(\mathrm{~s}, 3 \mathrm{H}, \mathrm{CH}_{3}\right) ;{ }^{13} \mathrm{C}-\mathrm{NMR}$ (DMSO- $\left.d_{6}, \mathrm{ppm}\right): \delta 197.46,166.30,153.57,151.81,150.17,138.54$, 138.06, 137.05, 136.88, 134.02, 129.69, 129.32, 128.51, 127.84, 122.58, 122.53, 115.99, 80.18, 41.74, 39.18, 21.70. MS (ESI) $m / z: 428.3\left([\mathrm{M}+\mathrm{H}]^{+}\right), 450.2\left([\mathrm{M}+\mathrm{Na}]^{+}\right) ; 86.0 \%$ ee as determined by HPLC (Daicel Chiralpak AD-H, hexane/ethanol $=50: 50$, flow rate $1.0 \mathrm{~mL} / \mathrm{min}, \lambda=254 \mathrm{~nm}$ ), $\mathrm{t}_{R}($ minor $)=8.02 \mathrm{~min}, \mathrm{t}_{R}$ (major) $=8.95 \mathrm{~min}$.

(-)-1-(2,4-Dichlorophenyl)-3-(4-((6-methylquinazolin-4-yl)oxy)phenyl)-4-nitrobutan-1-one [(-)-3d]: White solid; yield $38.0 \%$, m.p. $142-144{ }^{\circ} \mathrm{C} ;[\alpha]_{D}^{25}=-51.8\left(\mathrm{c}=0.85, \mathrm{CHCl}_{3}\right)$; IR $\left(\mathrm{KBr}, \mathrm{cm}^{-1}\right)$ v: 3450.0 $(\mathrm{C}-\mathrm{H}, \mathrm{Qu}-\mathrm{N}=\mathrm{CH}-\mathrm{N}=\mathrm{C}), 1699.3(\mathrm{C}=\mathrm{O}), 1653.0(\mathrm{C}=\mathrm{N}), 1581.8-1423.5(\mathrm{C}=\mathrm{C}$, benzene and Qu-ring), 781.2 (C-Cl); ${ }^{1} \mathrm{H}-\mathrm{NMR}$ (DMSO- $\left.d 6, \mathrm{ppm}\right): \delta 8.67$ (s, $\left.1 \mathrm{H}, \mathrm{Qu}-2-\mathrm{H}\right), 8.14$ (s, 1H, Qu-5-H), 7.91-7.86 (m, 2H, Ar'-3-H, Ar'-6-H), 7.74 (d, 1H, Qu-7-H, J=2.30 Hz), 7.67 (d, 1H, Qu-8-H, J=8.05 Hz), 7.58-7.56 
(m, 1H, Ar'-5-H), 7.49 (d, 2H, Ar-2-H, Ar-6-H, J=8.55 Hz), 7.29 (d, 2H, Ar-3-H, Ar-5-H, J = 8.60 Hz), 5.04-4.92 (m, 2H, - $\left.\mathrm{CH}_{2}-\mathrm{NO}_{2}\right), 4.10-4.03(\mathrm{~m}, 1 \mathrm{H}, \mathrm{CH}), 3.52\left(\mathrm{~d}, 2 \mathrm{H},-\mathrm{CH}_{2}-\mathrm{CO}, J=6.90 \mathrm{~Hz}\right) ;{ }^{13} \mathrm{C}-\mathrm{NMR}$ (DMSO-d6, ppm): $\delta 199.47,166.32,153.57,151.92,150.18,138.54,137.43,137.23,137.04,131.62$, 136.90, 131.20, 130.62, 129.73, 128.16, 127.85, 122.64, 122.55, 116.00, 79.83, 45.60, 39.29, 21.70. MS (ESI) $m / z: 496.2\left([\mathrm{M}+\mathrm{H}]^{+}\right), 518.2\left([\mathrm{M}+\mathrm{Na}]^{+}\right) ; 91.8 \%$ ee as determined by HPLC (Daicel Chiralpak $\mathrm{AD}-\mathrm{H}$, hexane/ethanol $=50: 50$, flow rate $1.0 \mathrm{~mL} / \mathrm{min}, \lambda=254 \mathrm{~nm}), \mathrm{t}_{R}($ minor $)=6.31 \mathrm{~min}$, $\mathrm{t}_{R}$ (major) $=6.78 \mathrm{~min}$.

(-)-1-(4-Chlorophenyl)-4-nitro-3-(4-(quinazolin-4-yloxy)phenyl)butan-1-one [(-)-3e]: White solid; yield $35.0 \%$, m.p. $146-148{ }^{\circ} \mathrm{C} ;[\alpha]_{D}^{25}=-56.2\left(\mathrm{c}=0.88, \mathrm{CHCl}_{3}\right) ; \mathrm{IR}\left(\mathrm{KBr}, \mathrm{cm}^{-1}\right)$ v: $3450.3(\mathrm{C}-\mathrm{H}$, $\mathrm{Qu}-\mathrm{N}=\mathrm{CH}-\mathrm{N}=\mathrm{C}), 1683.9(\mathrm{C}=\mathrm{O}), 1653.0(\mathrm{C}=\mathrm{N}), 1589.3-1489.1(\mathrm{C}=\mathrm{C}$, benzene and Qu-ring $), 700.1$ $(\mathrm{C}-\mathrm{Cl}) \mathrm{cm}^{-1}$; ${ }^{1} \mathrm{H}-\mathrm{NMR}$ (DMSO-d6, ppm): $\delta 8.72$ (s, 1H, Qu-2-H), 8.35 (d, 1H, Qu-8-H, $J=8.00 \mathrm{~Hz}$ ), $8.04\left(\mathrm{t}, 1 \mathrm{H}, \mathrm{Qu}-7-\mathrm{H}, J_{1}=8.60 \mathrm{~Hz}, J_{2}=6.85 \mathrm{~Hz}\right), 8.00$ (t, 3H, Qu-5-H, Ar'-2-H, Ar'-6-H, $J_{1}=3.45 \mathrm{~Hz}$, $\left.J_{2}=8.60 \mathrm{~Hz}\right), 7.79\left(\mathrm{t}, 1 \mathrm{H}, \mathrm{Qu}-6-\mathrm{H}, J_{1}=7.70 \mathrm{~Hz}, J_{2}=7.15 \mathrm{~Hz}\right), 7.61$ (d, 2H, Ar'-3-H, Ar'-5-H, $J=8.10 \mathrm{~Hz}), 7.51$ (d, 2H, Ar-2-H, Ar-6-H, $J=8.60 \mathrm{~Hz}), 7.30$ (d, 2H, Ar-3-H, Ar-5-H, $J=8.60 \mathrm{~Hz})$, 5.04-4.89 (m, 2H, - $\left.\mathrm{CH}_{2}-\mathrm{NO}_{2}\right), 4.14-4.10(\mathrm{~m}, 1 \mathrm{H}, \mathrm{CH}), 3.67-3.55\left(\mathrm{~m}, 2 \mathrm{H},-\mathrm{CH}_{2}-\mathrm{CO}\right) ;{ }^{13} \mathrm{C}-\mathrm{NMR}$ (DMSO-d6, ppm): $\delta 197.14,166.68,154.43,151.75,151.65,138.99,138.21,135.52,135.26,130.46$, $129.70,129.43,128.67,128.05,123.93,122.63,116.16,80.13,41.75,39.12$. MS (ESI) $m / z: 448.2$ $\left([\mathrm{M}+\mathrm{H}]^{+}\right), \quad 470.2\left([\mathrm{M}+\mathrm{Na}]^{+}\right) ; 99 \%$ ee as determined by HPLC (Daicel Chiralpak AD-H, hexane/ethanol $=50: 50$, flow rate $1.0 \mathrm{~mL} / \mathrm{min}, \lambda=254 \mathrm{~nm}), \mathrm{t}_{R}$ (major) $=9.61 \mathrm{~min}$.

(-)-1-(4-Methoxyphenyl)-4-nitro-3-(4-(quinazolin-4-yloxy)phenyl)butan-1-one [(-)-3f]: White solid; yield $42.2 \%$, m.p. $144-146{ }^{\circ} \mathrm{C} ;[\alpha]_{D}^{25}=-49.8\left(\mathrm{c}=0.88, \mathrm{CHCl}_{3}\right) ; \mathrm{IR}\left(\mathrm{KBr}, \mathrm{cm}^{-1}\right)$ v: $3450.3(\mathrm{C}-\mathrm{H}$, $\mathrm{Qu}-\mathrm{N}=\mathrm{CH}-\mathrm{N}=\mathrm{C}), 1683.9(\mathrm{C}=\mathrm{O}), 1653.0(\mathrm{C}=\mathrm{N}), 1589.3-1489.1(\mathrm{C}=\mathrm{C}$, benzene and Qu-ring $) ;{ }^{1} \mathrm{H}-\mathrm{NMR}$ (DMSO-d6, ppm): $\delta 8.73(\mathrm{~s}, 1 \mathrm{H}, \mathrm{Qu}-2-\mathrm{H}), 8.35(\mathrm{~d}, 1 \mathrm{H}, \mathrm{Qu}-8-\mathrm{H}, J=7.40 \mathrm{~Hz}), 8.03(\mathrm{t}, 1 \mathrm{H}, \mathrm{Qu}-7-\mathrm{H}$, $\left.J_{1}=8.60 \mathrm{~Hz}, J_{2}=6.85 \mathrm{~Hz}\right), 8.00(\mathrm{~d}, 1 \mathrm{H}, \mathrm{Qu}-5-\mathrm{H}, J=8.05 \mathrm{~Hz}), 7.96\left(\mathrm{t}, 1 \mathrm{H}, \mathrm{Qu}-6-\mathrm{H}, J_{1}=8.00 \mathrm{~Hz}\right.$, $J_{2}=6.85 \mathrm{~Hz}$ ), 7.51 (d, 2H, Ar'-3-H, Ar'-5-H, $J=8.60 \mathrm{~Hz}$ ), 7.30 (d, 2H, Ar-2-H, Ar-6-H, $J=8.60 \mathrm{~Hz}$ ), 7.05 (d, 2H, Ar-3-H, Ar-5-H, $J=8.60 \mathrm{~Hz}), 5.05-4.90\left(\mathrm{~m}, 2 \mathrm{H},-\mathrm{CH}_{2}-\mathrm{NO}_{2}\right), 4.15-4.09$ (m, 1H, CH), $3.85\left(\mathrm{~s}, 3 \mathrm{H}, \mathrm{CH}_{3}\right), 3.61-3.46\left(\mathrm{~m}, 2 \mathrm{H},-\mathrm{CH}_{2}-\mathrm{CO}\right) ;{ }^{13} \mathrm{C}-\mathrm{NMR}$ (DMSO-d6, ppm): $\delta 196.21,166.80$, $154.44,151.71,151.62,138.99,138.21,135.52$, 135.26, 130.46, 129.70, 129.43, 128.67, 128.05, 123.93, 122.63, 116.16, 80.13, 41.75, 39.12. MS (ESI) $\mathrm{m} / \mathrm{z}: 444.3\left([\mathrm{M}+\mathrm{H}]^{+}\right), 466.2\left([\mathrm{M}+\mathrm{Na}]^{+}\right) ; 92.0 \%$ $e e$ as determined by HPLC (Daicel Chiralpak AD-H, hexane/ethanol $=50: 50$, flow rate $1.0 \mathrm{~mL} / \mathrm{min}$, $\lambda=254 \mathrm{~nm}), \mathrm{t}_{R}($ minor $)=14.50 \mathrm{~min}, \mathrm{t}_{R}($ major $)=15.20 \mathrm{~min}$.

(-)-1-(4-Chlorophenyl)-3-(4-((6-methylquinazolin-4-yl)oxy)phenyl)-4-nitrobutan-1-one [(-)-3g]:White solid; yield 46.1\%, m.p. $210-212{ }^{\circ} \mathrm{C} ;[\alpha]_{D}^{25}=-9.8\left(\mathrm{c}=1.20, \mathrm{CHCl}_{3}\right)$; IR $\left(\mathrm{KBr}, \mathrm{cm}^{-1}\right)$ v: $3444.9(\mathrm{C}-\mathrm{H}$, $\mathrm{Qu}-\mathrm{N}=\mathrm{CH}-\mathrm{N}=\mathrm{C}), 1678.1(\mathrm{C}=\mathrm{O}), 1589.3(\mathrm{C}=\mathrm{N}), 1548.9-1496.8(\mathrm{C}=\mathrm{C}$, benzene and Qu-ring $), 814.0$ (C-Cl); ${ }^{1} \mathrm{H}-\mathrm{NMR}$ (DMSO-d6, ppm): $\delta 8.66$ (s, 1H, Qu-2-H), 8.14 (s, 1H, Qu-5-H), 8.00 (d, 2H, Ar'-2-H, Ar'-6-H, $J=4.00 \mathrm{~Hz}$ ), 7.88 (s, 2H, Qu-7-H, Qu-8-H), 7.62 (d, 2H, Ar'-3-H, Ar'-5-H, J=12.60 Hz), 7.51 (d, 2H, Ar-2-H, Ar-6-H, $J=4.55$ Hz), 7.28 (d, 2H, Ar-3-H, Ar-5-H, J=4.60 Hz), 5.04-4.90 (m, $\left.2 \mathrm{H},-\mathrm{CH}_{2}-\mathrm{NO}_{2}\right), 4.17-4.08(\mathrm{~m}, 1 \mathrm{H}, \mathrm{CH}), 3.67-3.55\left(\mathrm{~m}, 2 \mathrm{H},-\mathrm{CH}_{2}-\mathrm{CO}\right), 2.57\left(\mathrm{~s}, 3 \mathrm{H}, \mathrm{CH}_{3}\right) ;{ }^{13} \mathrm{C}-\mathrm{NMR}$ (DMSO-d6, ppm): $\delta 197.39,166.39,153.57,151.82,150.16,138.99,138.54,137.87,137.08,135.56$, 
130.80, 129.19, 127.84, 122.65, 122.53, 120.06, 116.13, 79.68, 42.01, 39.54, 21.71. MS (ESI) m/z: 462.2 $\left([\mathrm{M}+\mathrm{H}]^{+}\right), 484.2\left([\mathrm{M}+\mathrm{Na}]^{+}\right) ; 95.9 \%$ ee as determined by HPLC (Daicel Chiralpak AD-H, hexane/ethanol $=50: 50$, flow rate $1.0 \mathrm{~mL} / \mathrm{min}, \lambda=254 \mathrm{~nm}), \mathrm{t}_{R}($ minor $)=9.15 \mathrm{~min}, \mathrm{t}_{R}($ major $)=10.43 \mathrm{~min}$.

(-)-1-(2-Chlorophenyl)-3-(4-((6-methylquinazolin-4-yl)oxy)phenyl)-4-nitrobutan-1-one [(-)-3h]: White solid; yield 40.3\%, m.p. $67-69{ }^{\circ} \mathrm{C}$; $[\alpha]_{D}^{25}=-32.8 .4\left(\mathrm{c}=0.97, \mathrm{CHCl}_{3}\right)$; IR $\left(\mathrm{KBr}, \mathrm{cm}^{-1}\right)$ v: $3442.9(\mathrm{C}-\mathrm{H}$, $\mathrm{Qu}-\mathrm{N}=\mathrm{CH}-\mathrm{N}=\mathrm{C}), 1699.3(\mathrm{C}=\mathrm{O}), 1653.0(\mathrm{C}=\mathrm{N}), 1580.5-1498.7(\mathrm{C}=\mathrm{C}$, benzene and Qu-ring $), 752.2$ $(\mathrm{C}-\mathrm{Cl}) \mathrm{cm}^{-1}$; ${ }^{1} \mathrm{H}-\mathrm{NMR}$ (DMSO- $\left.d 6, \mathrm{ppm}\right): \delta 8.67$ (s, 1H, Qu-2-H), 8.14 (s, 1H, Qu-5-H), 7.92-7.87 (m, 2H, Qu-6-H, Ar'-6-H), 7.59 (d, 1H, Qu-7-H, J = 8.05 Hz), 7.56-7.53 (m, 2H, Ar'-3-H, Ar'-5-H), 7.49 (d, 2H, Ar-2-H, Ar-6-H, $J=8.60$ Hz), 7.47-7.44 (m, 1H, Ar'-4-H), 7.29 (d, 2H, Ar-3-H, Ar-5-H, $J=8.60 \mathrm{~Hz}), 5.05-4.93\left(\mathrm{~m}, 2 \mathrm{H},-\mathrm{CH}_{2}-\mathrm{NO}_{2}\right), 4.11-4.05(\mathrm{~m}, 1 \mathrm{H}, \mathrm{CH}), 3.57-3.47\left(\mathrm{~m}, 2 \mathrm{H},-\mathrm{CH}_{2}-\mathrm{CO}\right)$, $2.57\left(\mathrm{~s}, 3 \mathrm{H}, \mathrm{CH}_{3}\right) ;{ }^{13} \mathrm{C}-\mathrm{NMR}$ (DMSO- $\left.d 6, \mathrm{ppm}\right): \delta 200.48,166.39,153.58,152.02,150.18,138.76$, 138.55, 137.48, 137.05, 133.01, 131.02, 130.12, 129.74, 129.61, 127.96, 127.86, 122.64, 122.64, 116.00, 79.85, 45.72, 39.31, 21.70. MS (ESI) $\mathrm{m} / \mathrm{z}: 462.2\left([\mathrm{M}+\mathrm{H}]^{+}\right), 484.2\left([\mathrm{M}+\mathrm{Na}]^{+}\right) ; 96.0 \%$ ee as determined by HPLC (Daicel Chiralpak AD-H, hexane/ethanol $=50: 50$, flow rate $1.0 \mathrm{~mL} / \mathrm{min}, \lambda=254 \mathrm{~nm}$ ), $\mathrm{t}_{R}($ minor $)=29.56 \min , \mathrm{t}_{R}($ major $)=31.80 \mathrm{~min}$.

(-)-1-(2-Fluorophenyl)-3-(4-((8-methylquinazolin-4-yl)oxy)phenyl)-4-nitrobutan-1-one [(-)-3i]:White solid; yield $42.5 \%$, m.p. $123-125{ }^{\circ} \mathrm{C} ;[\alpha]_{D}^{25}=-43.9\left(\mathrm{c}=0.82, \mathrm{CHCl}_{3}\right)$; IR $\left(\mathrm{KBr}, \mathrm{cm}^{-1}\right)$ v: 3442.9 $(\mathrm{C}-\mathrm{H}, \mathrm{Qu}-\mathrm{N}=\mathrm{CH}-\mathrm{N}=\mathrm{C}), 1678.1(\mathrm{C}=\mathrm{O}), 1608.6(\mathrm{C}=\mathrm{N}), 1545.0-1483.3(\mathrm{C}=\mathrm{C}$, benzene and Qu-ring); ${ }^{1} \mathrm{H}-\mathrm{NMR}$ (DMSO-d6, ppm): $\delta 8.76(\mathrm{~s}, 1 \mathrm{H}, \mathrm{Qu}-2-\mathrm{H}), 8.18(\mathrm{~d}, 1 \mathrm{H}, \mathrm{Qu}-5-\mathrm{H}, J=8.00 \mathrm{~Hz}), 7.89(\mathrm{~d}, 1 \mathrm{H}$, Ar'-6-H, $J=7.45 \mathrm{~Hz}$ ), 7.82 (t, 1H, Ar'-4-H, $\left.J_{1}=7.40 \mathrm{~Hz}, J_{2}=7.45 \mathrm{~Hz}\right), 7.70-7.65$ (m, 2H, Qu-7-H, Qu-8-H), 7.51 (d, 2H, Ar-2-H, Ar-6-H, $J=8.55$ Hz), 7.39-7.33 (m, 2H, Ar'-3-H, Ar'-5-H), 7.30 (d, $2 \mathrm{H}, \mathrm{Ar}-3-\mathrm{H}, \mathrm{Ar}-5-\mathrm{H}, J=8.05 \mathrm{~Hz}), 5.07-4.94$ (m, 2H, $\left.-\mathrm{CH}_{2}-\mathrm{NO}_{2}\right), 4.16-4.11(\mathrm{~m}, 1 \mathrm{H}, \mathrm{CH}), 3.54(\mathrm{~d}, 2 \mathrm{H}$, $\left.-\mathrm{CH}_{2}-\mathrm{CO}, J=5.15 \mathrm{~Hz}\right), 2.69\left(\mathrm{~s}, 3 \mathrm{H}, \mathrm{CH}_{3}\right) ;{ }^{13} \mathrm{C}-\mathrm{NMR}$ (DMSO-d6, ppm): $\delta 195.83,167.00,160.78$, $153.57,151.85,150.63,138.01,136.31,135.92,134.91,130.80,129.65,128.10,125.40,122.64$, $121.45,117.58,117.40,116.00,79.99,46.21,39.03,17.69$. MS (ESI) $m / z: 446.3\left([\mathrm{M}+\mathrm{H}]^{+}\right), 484.2$ $\left([\mathrm{M}+\mathrm{Na}]^{+}\right) ; 92.5 \%$ ee as determined by HPLC (Daicel Chiralpak AD-H, hexane/ethanol = 50:50, flow rate $1.0 \mathrm{~mL} / \mathrm{min}, \lambda=254 \mathrm{~nm}), \mathrm{t}_{R}($ minor $)=27.99 \mathrm{~min}, \mathrm{t}_{R}($ major $)=29.44 \mathrm{~min}$.

\section{Conclusions}

In summary, we have developed and reported for the first time an efficient approach for enantioselective synthesis of (-)-1-phenyl-3-(4-((8-methylquinazolin-4-yl)oxy)phenyl)-4-nitrobutan-1-ones by employing an addition reaction catalyzed by a cinchona alkaloid-derived squaramide catalyst. The desired products were obtained with high enantioselectivities $(81 \%-99 \%)$.

\section{Acknowledgments}

We are grateful to the National Natural Science Foundation of China (No. 21362004) and Collaborative Innovation Center for Natural Products and Biological Drugs of Yunnan for supporting the project. 


\section{Author Contributions}

DYH conceived and designed the experiments. DDX and YX performed the experiments and analyzed the data; YX and YD tested all the ee values of the compounds; DYH analyzed the data and wrote the paper, JW revised the paper. All authors contributed to this study, read and approved the final manuscript.

\section{Conflicts of Interest}

The authors declare that they have no competing interests

\section{References}

1. Wu, J.Z.; Cheng, C.C.; Shen, L.L.; Wang, Z.K.; Wu, S.B.; Li, W.L.; Chen, S.H.; Zhou, R.P.; Qiu, P.H. Synthetic chalcones with potent antioxidant ability on $\mathrm{H}_{2} \mathrm{O}_{2}$-induced apoptosis in PC12 cells. Int. J. Mol. Sci. 2014, 15, 18525-18539.

2. Kumar, C.S.C.; Loh, W.S.; Ooi, C.W.; Quah, C.K.; Fun, H.K. Heteroarylchalcones: Design, synthesis, X-ray crystal structures and biological evaluation. Molecules 2013, 18, 12707-12724.

3. Hamada, N.M.M.; Sharshira, E.M. Synthesis and antimicrobial evaluation of some heterocyclic chalcone derivatives. Molecules 2011, 16, 2304-2312.

4. Nguyen, T.T.N.; Do, T.H.; Huynh, T.N.P.; Tran, C.D.T.; Thai, K.M. Synthesis and antibacterial activity of some heterocyclic chalcone analogues alone and in combination with antibiotics. Molecules 2012, 17, 6684-6696.

5. Hassan, S.Y. Synthesis, antibacterial and antifungal activity of some new pyrazoline and pyrazole derivatives. Molecules 2013, 18, 2683-2711.

6. Kang, J.E.; Cho, J.K.; Curtis-Long, M.J.; Ryu, H.W.; Kim, J.H.; Kim, H.J.; Yuk, H.J.; Kim, D.W.; Yuk, H.J.; Kim, D.W.; et al. Preparation of substituted pyridines and pyridazines with angiogenesis inhibiting activity for pharmaceutical use as antitumor agents. Molecules 2013, 18, 140-153.

7. Solomon, V.R.; Lee, H. Anti-breast cancer activity of heteroarylchalcone derivatives. Biomed. Pharmacother. 2012, 66, 213-220.

8. Kumar, D.; Kumar, N.M.; Akamatsu, K.; Kusaka, E.; Harada, H.; Ito, T. Synthesis and biological evaluation of indolylchalcones as antitumor agents. Bioorg. Med. Chem. Lett. 2010, 20, 3916-3919.

9. Domýngueza, J.N.; Charris, J.E.; Loboa, G.; de Domýnguezb, N.G.; Moreno, M.M.; Riggione, F.; Sanchez, E.; Olson, J.; Rosenthal, P.J. Synthesis of quinolinylchalcones and evaluation of their antimalarial activity. Eur. J. Med. Chem. 2001, 36, 555-560.

10. Hayat, F.; Moseley, E.; Salahuddin, A.; Zyl, R.L.V.; Azam, A. Antiprotozoal activity of chloro-quinoline based chalcones. Eur. J. Med. Chem. 2011, 46, 1897-1905.

11. Kotra, V.; Ganapathy, S.; Adapa, S.R. Synthesis of new quinolinylchalcones as anticancer and anti-inflammatory agents. Ind. J. Chem. 2010, 49B, 1109-1116.

12. Rizvi, S.U.F.; Siddiqui, H.L.; Johns, M.; Detorio, M.; Schinazi, R.F. Anti-HIV-1 and cytotoxicity studies of piperidyl-thienylchalcones and their 2-pyrazoline derivatives. Med. Chem. Res. 2012, 21, 3741-3749. 
13. Singh, P.; Anand, A.; Kumar, V. Recent developments in biological activities of chalcones: A minireview. Eur. J. Med. Chem. 2014, 85, 758-777.

14. Luo, J.; Jiang, C.H.; Wang, H.F.; Xu, L.W.; Lu, Y.X. Direct asymmetric Michael addition of phthalide derivatives to chalcones .Tetrahedron Lett. 2013, 54, 5261-5265.

15. Malerich, J.P.; Hagihara, K.; Rawal, V.H. Chiral squaramide derivatives are excellent hydrogen bond donor catalysts. J. Am. Chem. Soc. 2008, 130, 14416-14419.

16. Yang, W, Du, D.M. Highly enantioselective Michael addition of nitroalkanes to chalcones using chiral squaramides as hydrogen bonding organocatalysts. Org. Lett. 2010, 12, 5450-5453.

17. Herchl, R.; Waser, M. Asymmetric cyclopropanation of chalcones using chiral phase-transfer catalysts. Tetrahedron Lett. 2013, 54, 2472-2475.

18. Liu, Y.; Sun, B.F.; Wang, B.M. Catalytic asymmetric conjugate addition of simple alkyl thiols to $\alpha, \beta$-unsaturated $N$-acylated oxazolidin-2-oneswith bifunctional catalysts. J. Am. Chem. Soc. 2009, $131,418-419$.

19. Ye, Z.; Malerich, P.; Viresh, H. Squaramide-catalyzed enantioselective of diphenylphosphite to nitroalkenes. Angew. Chem. Int. Ed. 2010, 122, 157-160.

20. Xie, Y. Synthesis and Biological Activity of Chalcone Derivatives Containing Quinazoline Moiety. Master's Thesis, Guizhou University, Guiyang, China, 20 June 2013.

Sample Availability: Samples of the compounds are available from the authors.

(C) 2014 by the authors; licensee MDPI, Basel, Switzerland. This article is an open-access article distributed under the terms and conditions of the Creative Commons Attribution license (http://creativecommons.org/licenses/by/4.0/). 